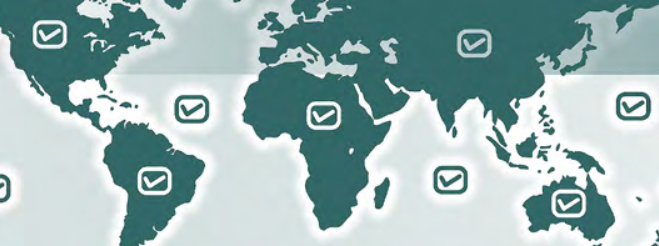

NOTES ON GEOGRAPHIC DISTRIBUTION

\title{
Chrotopterus auritus (Peters, 1856) (Chiroptera, Phyllostomidae): first record for the state of Rio Grande do Norte, northeastern Brazil
}

\author{
Gustavo Henrique Nunes Basílio ${ }^{1,5}$, Jan Pierre Martins de Araujo ${ }^{1}$, Juan Carlos Vargas Mena², \\ Patrício A. da Rocha ${ }^{3} \&$ Marcelo Augusto Freitas Kramer ${ }^{1,4}$
}

\begin{abstract}
${ }^{1}$ Sociedade Espeleológica Potiguar. CEP 59375-000, Rua da Matriz, Centro, Acari, Rio Grande do Norte, Brazil
${ }^{2}$ Departamento de Ecologia, Programa de Pós-Graduação em Ecologia, Universidade Federal do Rio Grande do Norte, Centro de Biociências Campus Lagoa Nova, 59072-970, Natal, Rio Grande do Norte, Brazil

${ }^{3}$ Programa de Pós-Graduação em Ciências Biológicas (Zoologia), CCEN, Universidade Federal da Paraíba, Campus I, 58051-900, João Pessoa, Paraíba, Brazil

${ }^{4}$ Department of Geosciences and Don Sundquist Center of Excellence in Paleontology, East Tennessee State University, Johnson City, TN 37614, USA

${ }^{5}$ Corresponding author. E-mail: gustavohenriquebiologia@gmail.com
\end{abstract}

\begin{abstract}
Chrotopterus auritus is a phyllostomid bat with a wide distribution in the Neotropics. It has been recorded in Brazil's 6 biomes but with few records in the Caatinga. We provide the first record of $C$. auritus for Rio Grande do Norte state, northeastern Brazil, based on records from 2 caves, Três Inchu and Gruta da Carrapateira. The nearest records are ca. $400 \mathrm{~km}$ southeast in Ceará state and ca. $350 \mathrm{~km}$ northwest in Pernambuco state. Our new records fill the northeastern distributional gap of C. auritus in Brazil and South America.
\end{abstract}

Key words. Bats; Chrotopterus; geographic distribution; Caatinga; caves

Chrotopterus auritus(Peters, 1856) is one the largest phyllostomid bats and the single representative of the genus Chrotopterus Peters, 1865 (Simmons 2005). As well as Vampyrum spectrum (Linnaeus, 1758) and Trachops cirrhosus (Spix, 1823), C. auritus is considered a predominantly carnivorous species (FERRAREZzI \& GimeneZ 1996) but occasionally including insects (MEDELLÍN 1988) and fruits (BERNARD 2002) in its diet. It is known to occur in southern Mexico, Central America, Colombia, Venezuela, Guyana, Surinam, French Guiana, Brazil, Ecuador, Peru, Bolivia, Paraguay, and northern Argentina (Williams \& Genoways 2008). In Brazil, this species has been recorded in all biomes (Paglia et al. 2012) but there are few records for the Caatinga (Fig. 1; Table 1). The Caatinga is restricted to Brazil and is a mosaic of tropical dry forest with xeric shrublands (LEAL et al. 2003). With a predominant semi-arid climate (MMA 2017) and located in the Northeast Region, the Caatinga has an area of over 800,000 $\mathrm{km}^{2}$, which represents about $11 \%$ of Brazil's territory, and covering 9 states and the northern part Minas Gerais. Herein, we provide the first occurrence record of Chrotopterus auritus for the state of Rio Grande do Norte, northeastern Brazil, based on records from 2 separate caves in the Caatinga on the west side of the state.

On 28 August 2015 at $23: 40 \mathrm{~h}$ an individual of $C$. auritus was observed and photographed in the Gruta da Carrapateira. Capture attempts were unsuccessful but the individual was identified through direct observation using binoculars and photographic records. The calcareous cave is located in the municipality of Felipe Guerra $\left(05^{\circ} 33^{\prime} 38.2^{\prime \prime} \mathrm{S}, 037^{\circ} 39^{\prime} 50.3^{\prime \prime} \mathrm{W}\right.$, altitude ca. $32 \mathrm{~m}$ ) about $348 \mathrm{~km}$ west of Natal and has 5 entrances and a linear development of $242.2 \mathrm{~m}$.

The second record is based on a colony of 8 individuals and an adult male captured with a hand net (Fig. 3) inside the Três Inchu cave $\left(06^{\circ} 03^{\prime} 07^{\prime \prime} \mathrm{S}, 037^{\circ} 54^{\prime} 03^{\prime \prime} \mathrm{W}\right)$ on 18 December 2015. The Três Inchu is a marble cave located in the municipality of Martins, ca. $362 \mathrm{~km}$ from Natal, with a linear development of $146.0 \mathrm{~m}$ and 5 recognized entrances, some of which are skylights, providing for photic zones inside.

The Três Inchu specimen was handled in accordance with Sikes et al. (2011), euthanized and fixed in $10 \%$ formaldehyde and preserved in $70 \%$ ethanol with subsequent extraction of the skull (Fig. 2). Cranial and external measurements were taken following Vizotto \& TADDEI (1973) using a digital caliper $(0.2 \mathrm{~mm})$. The specimen was collected under permit SISBIO/IBAMA 52492-1 and is deposited in the mammal collection of the Federal University of Sergipe (CMUFS) under voucher number CMUFS 259.

Our voucher of Chrotopterus auritus has the set of characters that distinguish this species from other members of the Phyllostominae, such as its large size (forearm $74-83 \mathrm{~mm}$ ), presence of 1 pair of lower incisors and 3 lower premolars (Fig. 2), rudimentary tail, calcar longer than the foot, large rounded ears not connected by a band, horseshoe of noseleaf cup-shaped and continuous with spear, and long thumb with a large strongly curved claw (Fig. 3) (Medellín 1989; WiLliams 


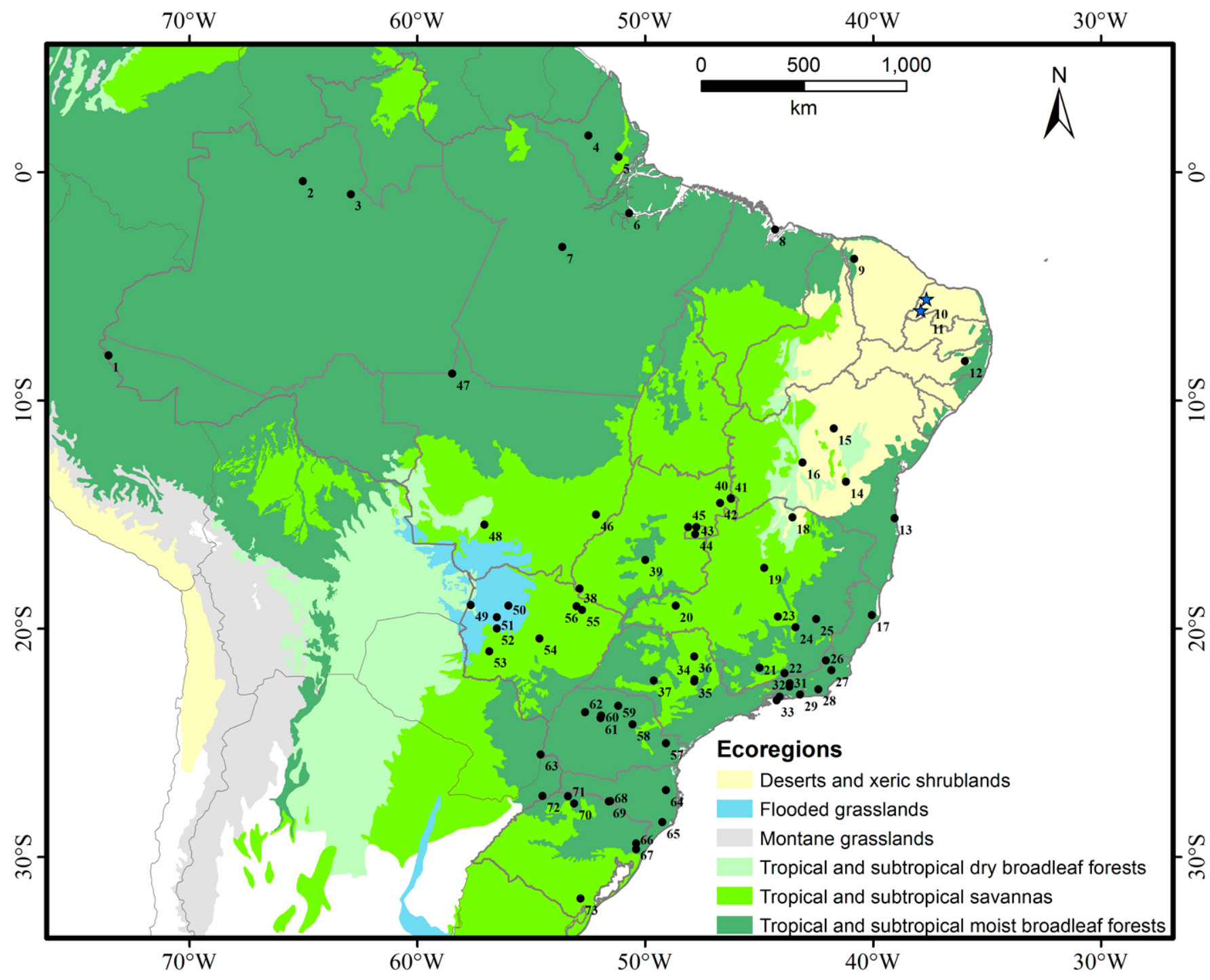

Figure 1. Records of Chrotopterus auritus in Brazil. The numbers corresponding to the records are indicated in Table 1. Blue stars represent the new records from the state of Rio Grande do Norte, Brazil.

Table 1. Locality records for Chrotopterus auritus in Brazil. The code numbers refer to the points shown in Figure 1.

\begin{tabular}{|c|c|c|c|c|c|}
\hline Code & Locality & State & Latitude (S) & Longitude (W) & References \\
\hline 1 & Parque Nacional da Serra do Divisor & $A C$ & $08^{\circ} 03^{\prime}$ & $073^{\circ} 55^{\prime}$ & NOGUEIRA (1999) \\
\hline 2 & Santa Isabel do Rio Negro & AM & $00^{\circ} 04^{\prime}$ & $065^{\circ} 01^{\prime}$ & MORATELLI et al. (2010) \\
\hline 3 & Barcelos & AM & $00^{\circ} 96^{\prime}$ & $062^{\circ} 91^{\prime}$ & MORATELLI et al. (2010) \\
\hline 4 & Parque Nacional Montanha do Tumucumaque & AP & $01^{\circ} 06^{\prime}$ & $052^{\circ} 48^{\prime}$ & MARTINS et al. (2006) \\
\hline 5 & Floresta Nacional do Amapá & AP & $00^{\circ} 66^{\prime}$ & $051^{\circ} 16^{\prime}$ & MARTINS et al. (2006) \\
\hline 6 & Melgaço & PA & $01^{\circ} 08^{\prime}$ & $050^{\circ} 07^{\prime}$ & MARQUES-AGUIAR et al. (2003) \\
\hline 7 & Uruará & PA & $03^{\circ} 28^{\prime}$ & $053^{\circ} 63^{\prime}$ & CAJAIBA (2014) \\
\hline 8 & São Luiz & MA & $02^{\circ} 51^{\prime}$ & $044^{\circ} 03^{\prime}$ & CRUz et al. (2007) \\
\hline 9 & Ubajara & $\mathrm{CE}$ & $03^{\circ} 08^{\prime}$ & $040^{\circ} 83^{\prime}$ & UIEDA et al. (1980) \\
\hline 10 & Gruta da Carrapateira cave, Felipe Guerra & RN & $05^{\circ} 55^{\prime}$ & $037^{\circ} 65^{\prime}$ & This study \\
\hline 11 & Três Inchu cave, Martins & RN & $06^{\circ} 05^{\prime}$ & $037^{\circ} 09^{\prime}$ & This study \\
\hline 12 & $\begin{array}{l}\text { Ecológico Municipal Professor João Vasconcelos } \\
\text { Sobrinho, Caruaru }\end{array}$ & $\mathrm{PE}$ & $08^{\circ} 28^{\prime}$ & $035^{\circ} 96^{\prime}$ & SousA et al. (2004) \\
\hline 13 & Una & BA & $15^{\circ} 16^{\prime}$ & $039^{\circ} 05^{\prime}$ & FARIA et al. (2006) \\
\hline 14 & Chapada Diamantina & BA & $13^{\circ} 56^{\prime}$ & $041^{\circ} 18^{\prime}$ & GREGORIN \& MENDES (1999) \\
\hline 15 & Chapada Diamantina & BA & $11^{\circ} 23^{\prime}$ & $041^{\circ} 71^{\prime}$ & SBRAGIA \& CARDOSO (2008) \\
\hline 16 & Médio São Francisco, Paratinga & BA & $12^{\circ} 73^{\prime}$ & $043^{\circ} 09^{\prime}$ & SÁ-NETO \& MARINHO-FILHO (2013) \\
\hline 17 & Linhares & ES & $19^{\circ} 41^{\prime}$ & $040^{\circ} 05^{\prime}$ & PERACCHI \& ALBUQUERQUE (1993) \\
\hline
\end{tabular}


Table 1. Continued.

\begin{tabular}{|c|c|c|c|c|c|}
\hline Code & Locality & State & Latitude (S) & Longitude (W) & References \\
\hline 18 & Jaíba & MG & $15^{\circ} 13^{\prime}$ & $043^{\circ} 54^{\prime}$ & NOGUEIRA et al. (2015) \\
\hline 19 & Piarapora & MG & $17^{\circ} 35^{\prime}$ & $044^{\circ} 78^{\prime}$ & TAVARES et al. (2010) \\
\hline 20 & Uberlândia & MG & $19^{\circ} 00^{\prime}$ & $048^{\circ} 66^{\prime}$ & PEDRO \& TADDEI (2014) \\
\hline 21 & São Tomé das Letras & MG & $21^{\circ} 71^{\prime}$ & $044^{\circ} 98^{\prime}$ & TAVARES et al. (2010) \\
\hline 22 & Serra Negra & MG & $21^{\circ} 96^{\prime}$ & $043^{\circ} 88^{\prime}$ & NOBRE et al. (2009) \\
\hline 23 & Sete Lagoas & MG & $19^{\circ} 84^{\prime}$ & $044^{\circ} 18^{\prime}$ & TAVARES et al. (2010) \\
\hline 24 & Santa Barbara & MG & $19^{\circ} 95^{\prime}$ & $043^{\circ} 04^{\prime}$ & TAVARES et al. (2010) \\
\hline 25 & Serra da Mantiqueira & MG & $19^{\circ} 58^{\prime}$ & $042^{\circ} 05^{\prime}$ & STALLINGS et al. (1990) \\
\hline 26 & Paraíso do Tobias, Miracema & RJ & $21^{\circ} 04^{\prime}$ & $042^{\circ} 06^{\prime}$ & ESBÉRARD et al. (2010) \\
\hline 27 & Parque Estadual Desengano & RJ & $21^{\circ} 83^{\prime}$ & $041^{\circ} 83^{\prime}$ & MODESTO et al. (2008) \\
\hline 28 & Silva Jardim & RJ & $22^{\circ} 66^{\prime}$ & $042^{\circ} 39^{\prime}$ & BAPTISTA \& MELLO (2001) \\
\hline 29 & Pedra Branca & RJ & $22^{\circ} 09^{\prime}$ & $043^{\circ} 02^{\prime}$ & DIAS et al. (2002) \\
\hline 30 & Engenheiro Paulo de Frontin & RJ & $22^{\circ} 55^{\prime}$ & $043^{\circ} 66^{\prime}$ & DIAS et al. (2010) \\
\hline 31 & Vassouras & RJ & $22^{\circ} 04^{\prime}$ & $043^{\circ} 65^{\prime}$ & DIAS et al.(2002) \\
\hline 32 & Mangaratiba & RJ & $22^{\circ} 98^{\prime}$ & $044^{\circ} 01^{\prime}$ & LUZ et al. (2011) \\
\hline 33 & Ilha Grande - Angra dos Reis & RJ & $23^{\circ} 15^{\prime}$ & $044^{\circ} 22^{\prime}$ & ESBÉRARD et al. (2006) \\
\hline 34 & Itaperina & SP & $22^{\circ} 23^{\prime}$ & $047^{\circ} 83^{\prime}$ & UIEDA et al. (2007) \\
\hline 35 & Estação Experimental de Itirapina & SP & $22^{\circ} 32^{\prime}$ & $047^{\circ} 84^{\prime}$ & SATO et al. (2015) \\
\hline 36 & Ribeirão Preto & SP & $21^{\circ} 22^{\prime}$ & $047^{\circ} 84^{\prime}$ & PAssos et al. (2003) \\
\hline 37 & Estação Ecológica dos Caetetus, Gália & SP & $22^{\circ} 27^{\prime}$ & $049^{\circ} 61^{\prime}$ & PEDRO et al. (2014) \\
\hline 38 & Parque Nacional das Emas & GO & $18^{\circ} 25^{\prime}$ & $052^{\circ} 88^{\prime}$ & RODRIGUES et al. (2002) \\
\hline 39 & Indiara & GO & $17^{\circ} 00^{\prime}$ & $050^{\circ} 00^{\prime}$ & SILVA et al. (2011) \\
\hline 40 & Posse & GO & $14^{\circ} 31^{\prime}$ & $046^{\circ} 23^{\prime}$ & ESBÉRARD et al. (2001) \\
\hline 41 & APA Nascente Rio Vermelho & GO & $14^{\circ} 28^{\prime}$ & $046^{\circ} 25^{\prime}$ & ESBÉRARD et al. (2005) \\
\hline 42 & Alvorada do Norte & GO & $14^{\circ} 05^{\prime}$ & $046^{\circ} 71^{\prime}$ & BEZERRA \& MARINHO-FILHO (2010) \\
\hline 43 & Planaltina & DF & $15^{\circ} 55^{\prime}$ & $047^{\circ} 75^{\prime}$ & BREDT \& MAGALHAES (1999) \\
\hline 44 & Paranoá & DF & $15^{\circ} 86^{\prime}$ & $047^{\circ} 08^{\prime}$ & BREDT \& MAGALHAES (1999) \\
\hline 45 & Brazlândia & DF & $15^{\circ} 55^{\prime}$ & $048^{\circ} 11^{\prime}$ & BREDT \& MAGALHAES (1999) \\
\hline 46 & Near to Nova Xavantina & MT & $15^{\circ} 01^{\prime}$ & $052^{\circ} 15^{\prime}$ & OLIVEIRA et al. (2015) \\
\hline 47 & Cedro Trail, lower Juruena River & MT & $08^{\circ} 83^{\prime}$ & $058^{\circ} 46^{\prime}$ & DALPONTE et al. (2016) \\
\hline 48 & Estação Ecologica Serra das Araras & MT & $15^{\circ} 45^{\prime}$ & $057^{\circ} 05^{\prime}$ & GONÇALVES \& GREGORIN (2004) \\
\hline 49 & Corumbá & MS & $18^{\circ} 96^{\prime}$ & $057^{\circ} 65^{\prime}$ & BORDIGNON \& FRANÇA (2004) \\
\hline 50 & Nhecolândia & MS & $19^{\circ} 00^{\prime}$ & $056^{\circ} 00^{\prime}$ & OLIVEIRA et al. (2011) \\
\hline 51 & Abobral & MS & $19^{\circ} 05^{\prime}$ & $056^{\circ} 5^{\prime}$ & OLIVEIRA et al. (2011) \\
\hline 52 & Miranda & MS & $20^{\circ} 00^{\prime}$ & $056^{\circ} 05^{\prime}$ & OLIVEIRA et al. (2011) \\
\hline 53 & Parque Nacional Serra da Bodoquena & MS & $20^{\circ} 99^{\prime}$ & $056^{\circ} 83^{\prime}$ & CAMARGO et al. (2009) \\
\hline 54 & Campo Grande & MS & $20^{\circ} 43^{\prime}$ & $054^{\circ} 63^{\prime}$ & BORDIGNON (2005) \\
\hline 55 & Chapadão do Sul & MS & $19^{\circ} 18^{\prime}$ & $052^{\circ} 76^{\prime}$ & BORDIGNON et al. (2006) \\
\hline 56 & Paraíso das Águas & MS & $19^{\circ} 02^{\prime}$ & $053^{\circ} 01^{\prime}$ & DALPONTE et al. (2016) \\
\hline 57 & Campinhos & PR & $25^{\circ} 03^{\prime}$ & $049^{\circ} 08^{\prime}$ & ARNONE \& PASSOS (2003) \\
\hline 58 & Telêmaco Borba & PR & $24^{\circ} 02^{\prime}$ & $050^{\circ} 55^{\prime}$ & REIS et al. (1999) \\
\hline 59 & Londrina & PR & $23^{\circ} 38^{\prime}$ & $051^{\circ} 18^{\prime}$ & REIS et al. (2003) \\
\hline 60 & Sâo Pedro do Ivaí & PR & $23^{\circ} 81^{\prime}$ & $051^{\circ} 93^{\prime}$ & CARNEIRO (2008) \\
\hline 61 & Fênix & PR & $23^{\circ} 91^{\prime}$ & $051^{\circ} 95^{\prime}$ & BIANCONI et al. (2004) \\
\hline 62 & Cianorte & PR & $23^{\circ} 66^{\prime}$ & $052^{\circ} 63^{\prime}$ & ORTÊNCIO-FILHO \& REIS (2009) \\
\hline 63 & Foz do Iguaçú & PR & $25^{\circ} 51^{\prime}$ & $054^{\circ} 58^{\prime}$ & GRACIOLLI \& CARVALHO (2001) \\
\hline 64 & Blumenau & SC & $27^{\circ} 08^{\prime}$ & $049^{\circ} 08^{\prime}$ & ALTHOFF (2007) \\
\hline 65 & Pedras Grandes & SC & $28^{\circ} 48^{\prime}$ & $049^{\circ} 25^{\prime}$ & CARVALHO et al. (2013) \\
\hline 66 & Vale do Taquari & RS & $29^{\circ} 66^{\prime}$ & $050^{\circ} 04^{\prime}$ & KASPER et al. (2007) \\
\hline 67 & São Francisco de Paula & RS & $29^{\circ} 41^{\prime}$ & $050^{\circ} 04^{\prime}$ & MARQUES et al. (2011) \\
\hline 68 & Barracão & RS & $27^{\circ} 56^{\prime}$ & $051^{\circ} 53 \mathrm{~W}$ & WITT et al. (2010) \\
\hline 69 & Machadinho & RS & $27^{\circ} 58^{\prime}$ & $051^{\circ} 06^{\prime}$ & WITT et al. (2010) \\
\hline 70 & Rio Grande do Sul & RS & $27^{\circ} 66^{\prime}$ & $053^{\circ} 11^{\prime}$ & Peters et al. (2010) \\
\hline 71 & Frederico Westphalen & RS & $27^{\circ} 33^{\prime}$ & $053^{\circ} 38^{\prime}$ & BERNARDI et al. (2009) \\
\hline 72 & Derrubados & RS & $27^{\circ} 33^{\prime}$ & $054^{\circ} 05^{\prime}$ & KASPER et al. (2007) \\
\hline 73 & Rio Grande & RS & $31^{\circ} 49^{\prime}$ & $052^{\circ} 49^{\prime} \mathrm{W}$ & QUINTELA et al. (2011) \\
\hline
\end{tabular}




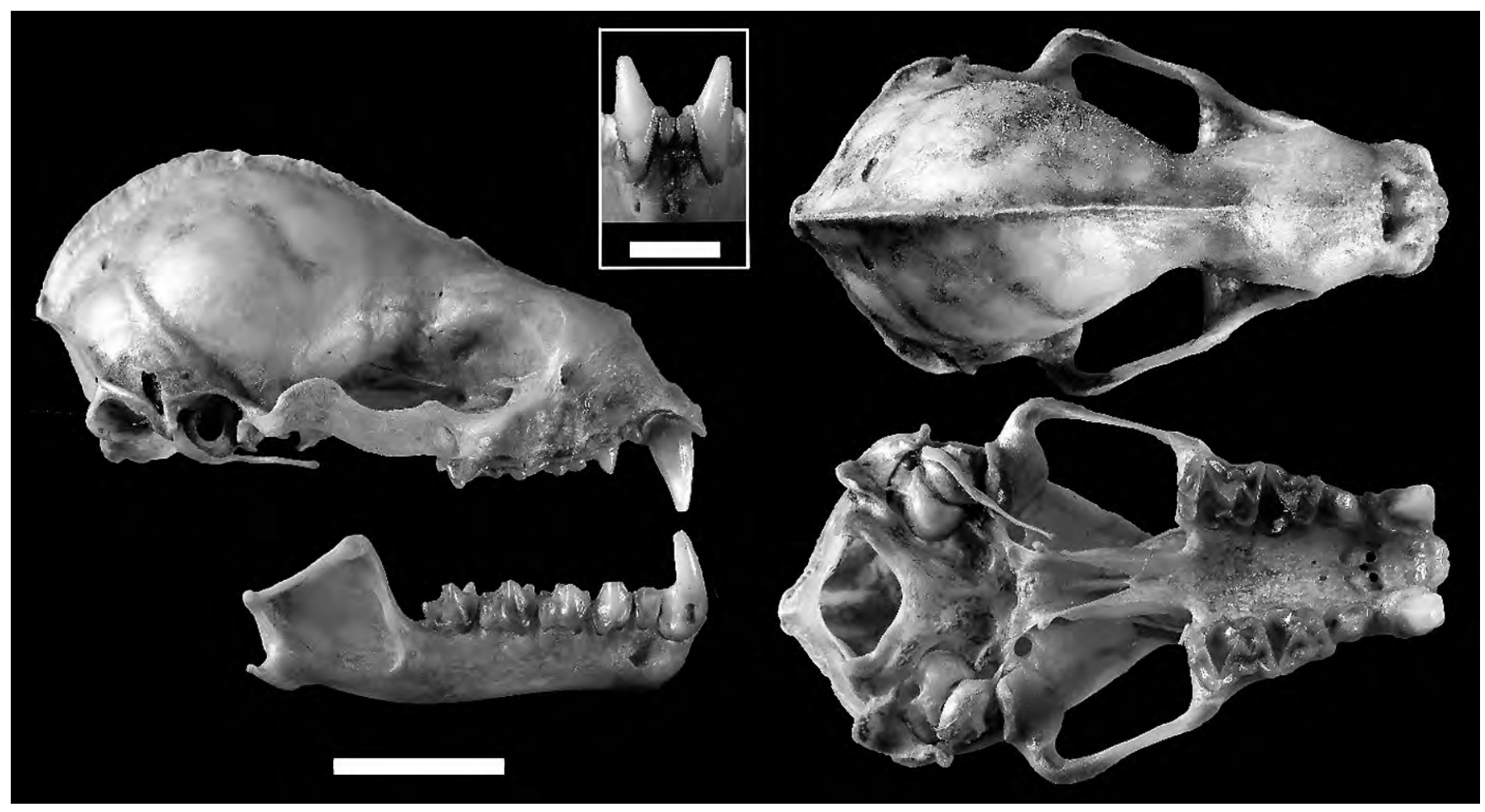

Figure 2.Dorsal, ventral and lateral views of the skull and lateral view of the mandible (scale bar=10 mm) of Chrotopterus auritus (CMUFS 259 ) from Três Inchu cave, Martins municipality, Rio Grande do Norte state, northeast Brazil. In detail, front view of the mandible showing the presence of 2 incisors (scale bar $=5 \mathrm{~mm}$ ).

\section{\& GenOWAYs 2008).}

Cranial and external measurements of the voucher, in millimeters, are: forearm length (84.6), hind foot length (21.1), calcar (22.3), ear (36.2), greatest length of skull (37.12), condylobasal length (32.41), breadth of braincase (14.28), post-orbital constriction (6.06), breadth across upper molars (12.18), breadth across upper canines (7.92), length of mandible (24.78) and length of maxillary tooth row (15.18). These measurements are within the known range for the species (see MedelLín 1988). In the case of the individual from Gruta da Carrapateira, not captured, it was possible to see external diagnostic characters of $C$. auritus, such as the cup-shaped horseshoe of the noseleaf and the long thumbs with large claw.

Our records of Chrotopterus auritus from Rio Grande do Norte fill the distributional gap of this species in northeastern
Brazil. They extend the distribution of C. auritus in ca. $400 \mathrm{~km}$ southeast and $350 \mathrm{~km}$ northwest from the Ceará (UIEDA et al. 1980) and Pernambuco (SousA et al. 2004) states, respectively.

The presence of $C$. auritus within the Caatinga may be unusual, since there are only 6 previous records from this biome (Fig. 1; Table 1). The records are: in xeric-woodlands of Caatinga (SÁ-Neto \& Marinho-Filho 2013); in Chapada Diamantina National Park in Bahia state (Gregorin \& Mendes 1999; Sbragia \& Cardoso 2008); in transition areas of Caatinga and the Amazon (UIEDA et al. 1980); in humid forest enclaves within the Caatinga known as "Brejos de Altitude" (Sousa et al. 2004); and in the southern limit of the Caatinga in Jaíba, northern Minas Gerais state (NogueIRA et al.2015). Moreover, 4 of these records were obtained from underground cavities (Gregorin \& Mendes 1999; Sbragia \&
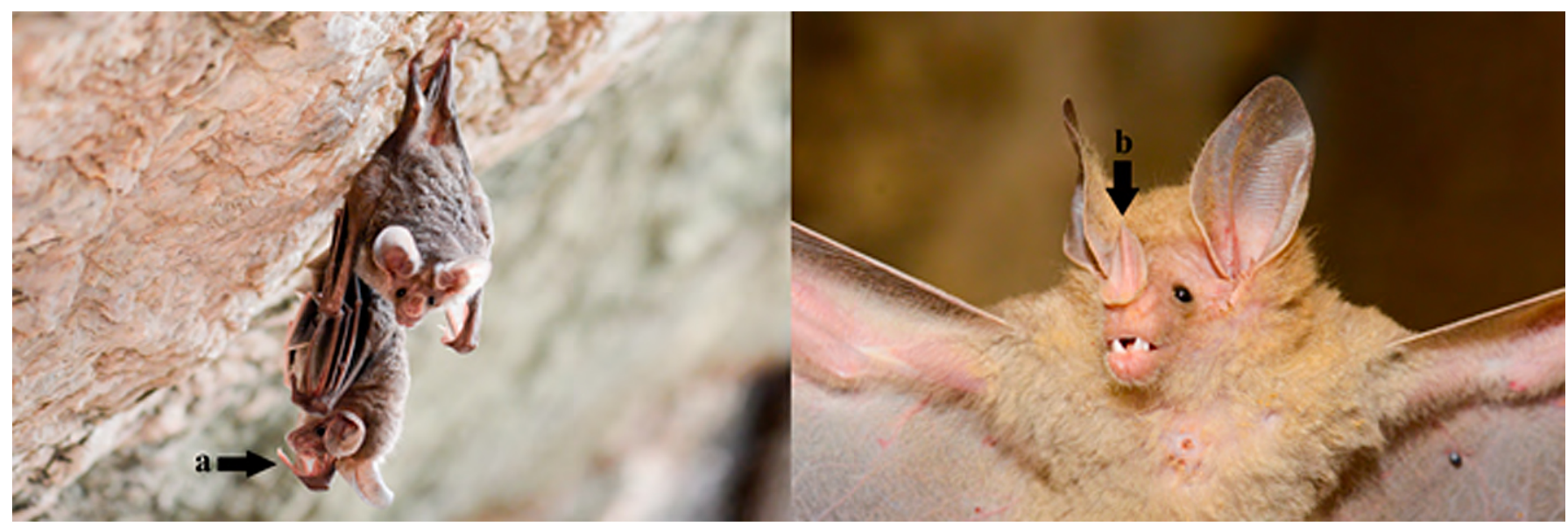

Figure 3. Chrotopterus auritus photographed at the Gruta Três Inchu, Martins municipality, Rio Grande do Norte state, northeast Brazil. Note the long thumb (arrow a) with a large strongly curved claw and the cup-shaped horseshoe of noseleaf (arrow b). 
Cardoso 2008; Uieda et al.1980; Nogueira et al. 2015).

Chrotopterus auritus is known to roostin caves, abandoned mines, buildings, and hollow trees (REID 2009). It inhabits mature evergreen forest and deciduous forests, but records in this latter formation are less common (MEDELLín 1989). Because $C$. auritus is a top predator, more regularly found in undisturbed habitats, its presence is considered an indicator of healthy forests (MedelLín 1989; GorRENSEN \& WiLliG 2004). Individuals generally form small groups from 3 to 6 individuals in their roost (ARITA \& VARGAS 1995; BREDT et al. 1999). When roosting in caves, this species is commonly found in cohabitation with other bat species (CoImBRA et al. 1982; Trajano 1985; Bredt et al. 1999). We observed C. auritus cohabitating with Desmodus rotundus (E. Geoffroy, 1810) and Peropteryx macrotis (Wagner, 1843) in Três Inchu cave and with Artibeus planirostris (Spix, 1823), Furipterus horrens(F. Cuvier, 1828), Desmodus rotundus, Diphylla ecaudata Spix, 1823, Glossophaga soricina (Pallas, 1766) and P. macrotis in Gruta da Carrapateira.

Caves in the Caatinga, including those in the state of Rio Grande do Norte, may be important roosting sites for $C$. auritus, and the presence of a high density of caves in the landscape may be a good predictor of a potential occurrence of this species. Furthermore, roosting in caves may provide benefits to $C$. auritus besides providing stable roost conditions. The cohabitation with others bats inside caves may allow for the predation of other smaller bats, a behavior already reported for C. auritus (BORDIGNON 2005; WitT \& FABIAN 2010).

Rio Grande do Norte is one of the least known states in relation to its chiropterofauna, with only 11 species currently recorded (Desmodus rotundus, Diphylla ecaudata, Lonchorhina aurita Tomes, 1863, Artibeus planirostris, Trachops cirrhosus, Natalus macrourus (Gervais, 1856), Peropteryx macrotis, Dermanura cinerea (Gervais, 1856), Molossus molossus (Pallas, 1766), Myotis nigricans (Schinz, 1821), Glossophaga soricina; OliveIra et al. 2003; FeIJó \& Nunes 2010; FERreira et al. 2010; BARros 2014). With 949 cavities currently cataloged, the state is fourth in Brazil for the number of natural underground cavities (CECAV/ICMBio 2016), which constitute potential important roost for the bat communities in the Caatinga of the state.

Anincrease of bat inventories in Rio Grande do Norte, both in caves as well as in other habitats, should provide a better understanding of the distribution of $C$. auritus and more generally the species richness in this state and in the Caatinga biome.

\section{ACKNOWLEDGEMENTS}

We thank the Sociedade Espeleológica Potiguar (SEP) for providing our main source of funding, the Universidade Federal de Sergipe (UFS) and the Universidade Federal do Rio Grande do Norte (UFRN) for supportingour study. We also thankSolon Rodrigues de Almeida Neto, Aldo Guimarães, Eugenia Cordero Schmidt, and Valtenci Santana for field assistance and Blaine W. Schubert for helping improve the manuscript. JCVM thanks the National Council for Scientific and Technological Development $(\mathrm{CNPq})$ for a research grant (Pes- quisador Visitante Especial-PVE "Ecologia e Conservação de Morcegosna Caatinga Potiguar" Project: 401467/2014-7); CAPES for the post-graduation scholarship, and the Wildlife Conservation Society (WCS) of Brazil for the help withfield logistics. PAR thanks CNPq for a research grant (processes 501701/2013-3 and 150407/2015-7).

\section{LITERATURE CITED}

Arita, H.T. \& J.A. Vargas. 1995. Natural history, interspecific association, and incidence of the cave bats of Yucatan, Mexico. The Southwestern Naturalist 40(1): 29-37. http://www.jstor.org/ stable/30054390

Arnone, I.S. \& F.C. Passos. 2007. Estrutura de comunidade da quiropterofauna (Mammalia, Chiroptera) do Parque Estadual de Campinhos, Paraná, Brasil. Revista Brasileira de Zoologia 24(3): 573-581. https://doi.org/10.1590/0031-1049.2015.55.01

Baptista, M. \& M. Mello. 2001. Preliminary inventory of the bat species of the Poço das Antas Reserve, RJ. Chiroptera Neotropical 7(1-2):133-135. http://chiropteraneotropical.net/index.php/cn/ article/view/123

Barros, M.A. 2014. First record of Molossusmolossus (Pallas, 1766) (Mammalia: Chiroptera) in the state of Rio Grande do Norte, northeastern Brazil. Check List 10(6): 1520-1524. https://doi. org $/ 10.15560 / 10.6 .1520$

Bernard, E. 2002. Diet, activity and reproduction of bats species (Mammalia, Chiroptera) in Central Amazonia, Brazil. Revista Brasileira de Zoologia 19(1):173-188. https://doi.org/10.1590/S1181752002000100016

Bernardi, I.P., J.M. D. Miranda, J. Sponchiado, E. Grotto, F.F. Jacomassa, E.M. Texeira, S.H. Roani \& F.C. Passos. 2009. Morcegos de Frederico Westphalen, Rio Grande do Sul, Brasil (Mammalia: Chiroptera): riqueza e utilização de abrigos. Biota Neotropica 9(3): 349-354. http://www.biotaneotropica.org.br/v9n3/ pt/abstract?article+bn03009032009

Bezerra, A.M. and J. Marinho-Filho. 2010. Bats of the Paranãriver valley, Tocantins and Goiás states, central Brazil. Zootaxa 2725: $41-56$.

Bianconi, G.V., S.B. Mikich \& W.A. Pedro. 2004. Diversidade de morcegos (Mammalia, Chiroptera) em remanescentes florestais do município de Fênix, noroeste do Paraná, Brasil. Revista Brasileira de Zoologia 21(4): 943-954. https://doi.org/10.1590/ S0101-81752004000400032

Bordignon, M. O. 2005. Predação de morcegos por Chrotopterus auritus (Peters, 1856) (Mammalia, Chiroptera) no Pantanal de Mato Grosso do Sul, Brasil. Revista Brasileira de Zoologia 22(4): 1207-1208. https://doi.org/10.1590/S0101-81752005000400058

Bordignon, M.O. 2006. Diversidade de morcegos (Mammalia, Chiroptera) do Complexo Aporé-Sucuriú, Mato Grosso do Sul, Brasil. Revista Brasileira de Zoologia 23(4): 1002-1009. https:// doi.org/10.1590/S0101-81752006000400004

Bordignon, M.O. \& A.O. FrançA. 2004. Análise preliminar sobre a diversidade de morcegos no Maciço do Urucum, Mato Grosso do Sul, Brasil. IV Simpósio sobre os recursos naturais e sócioeconômicos do Pantanal. Corumbá: Embrapa. http://www.cpap. embrapa.br/agencia/simpan/sumario/artigos/asperctos/pdf/ bioticos/C\%A2pia\%20de\%20604RBBordignon\%20OKVisto.pdf Bordignon, M.O., N.C. Cáceres, A.O. França, J. Casella \& C.F. VARGaS. 2006. Inventário da Mastofauna no Complexo Aporé-Sucuriú; pp. 129-142, in: T.C.S. Pagotto \& P.R. Souza (eds.). Biodiversidade do complexo Aporé-Sucuriú: subsídios à conservação e manejo do bioma Cerrado. Campo Grande: Editora da UFMS.

Bredt, A., W. Uieda \& E.D. Magalhães. 1999. Morcegos cavernícolas da região do Distrito Federal, centro-oeste do Brasil. Revista 
Brasileira de Zoologia 16(3): 731-770. https://doi.org/10.1590/ S0101-81751999000300012

Cajaiba, R.L. 2014. Morcegos (Mammalia, Chiroptera) em cavernas no município de Uruará, Pará, norte do Brasil. Biota Amazônia 4(1): 81-86. http://dx.doi.org/10.18561/2179-5746/biotaamazonia. v4n1p81-86

Camargo, G., E. Fischer, F. Gonçalves, G. Fernandes \& S. FerREIRA. 2009. Morcegos do Parque Nacional da Serra da Bodoquena, Mato Grosso do Sul, Brasil. Chiroptera Neotropical 15(1): 417-424. https://doi.org/10.1111/j.0030-1299.2004.12884.x

Carvalho, F., M.E. Fabian \& J.O. Menegheti. 2013. Vertical structure of an assemblage of bats (Mammalia: Chiroptera) in a fragment of Atlantic Forest in Southern Brazil. Zoologia 30(5): 491-498. https://doi.org/10.1590/S1984-46702013000500004

CECAV/ICMBio. 2016. Centro Nacional de Pesquisa e Conservação de Cavernas. Base de Dados Geoespacializados de Cavidades Naturais Subterrâneas do CECAV. Accessed at http://www.ibama. gov.br/cecav/\%20index.php?Id menu=228, 28 October 2016.

Coimbra Jr, C. E. A., M. M Borges, D.Q. Guerra, \& D.A. Mello. 1982. Contribuição à zoogeografia e ecologia de morcegos em regiões de cerrado do Brasil Central. Boletim Técnico Revista Brasil Florestal 7: 34-38.

Cruz, L.D., C. Martínez \& F.R. Fernandes. 2007. Comunidades de morcegos em hábitats de uma Mata Amazônica remanescente na Ilha de São Luís, Maranhão. Acta Amazonica 37(4): 613-619. https://doi.org/10.1590/S0044-59672007000400017

Dalponte, J.C., R. Gregorin, V.A. Esteves-Costa, E.C. Rocha \& R. MARCELinho. 2016. Bat survey of the lower Juruena River and five new records for the state of Mato Grosso, Brazil. Acta Amazonica 46(2): 227-232. https://doi.org/10.1590/1809-4392201500888

Dias, D., A.L. Peracchi, \& S.S.P Da Silva. 2002. Quirópteros do Parque Estadual da Pedra Branca, Rio de Janeiro, Brasil (Mammalia, Chiroptera). Revista Brasileira de Zoologia 19(2): 113-140. https://doi.org/10.1590/S0101-81752002000600012

Dias, D., S.N. Pereira, A.C. MaAs, M.A. Martins, M.A., D.P. Bolzan \& A.L. PeracChI. 2010. Quirópteros das regiões CentroSul e Médio Paraíba do estado do Rio de Janeiro (Mammalia, Chiroptera). Chiroptera Neotropical 16(1): 579-585. http:// chiropteraneotropical.net/index.php/cn/article/view/231

Esbérard, C. E.L., J. A. Motta, E. M. Calvo, V. M. Ferreira, J. C., Carvalho, C.C. Carvalho, C.R.P. Souza, E.A. Pires, G.M.V. Rosa, J.S. Reis, J.N. Araújo, K.E. Quege. 2001. Morcegos Cavernícolas de Mambaí e Arredores, Goiás, Brasil. Brasília:26vo Congresso Brasileiro de espeleologia. http://www. sbe.com.br/anais26cbe/26CBE 361-364.pdf

Esbérard, C.E.L., J.A. Motta \& C. Perigo. 2005. Morcegos cavernícolas da Área de Proteção Ambiental (APA) Nascentes do Rio vermelho, Goiás. Revista Brasileira de Zoociências 7(2): 311-325. https://zoociencias.ufjf.emnuvens.com.br/zoociencias/ article/view/165

Esbérard, C.E. L., T. Jordão-Nogueira, J.L. Luz, G.G.S. Melo, R. Mangolin, N. JucÁ, D.S.L. Raíces, M.C. Enrici \& H.G. Bergallo. 2006. Morcegos da Ilha Grande, Angra dos Reis, RJ, Sudeste do Brasil. Revista Brasileira de Zoociências 8(2): 147-153. https://zoociencias.ufjf.emnuvens.com.br/zoociencias/article/ view/110

Esbérard, C.E.L., M. Baptista, L.D. M. Costa, J.L. Luz \& E.C. Lourenço. 2010. Bats from Paraíso do Tobias, Northwest of Rio de Janeiro, Brazil. Biota Neotropica 10(4): 249-255. https://doi. org/10.1590/S1676-06032010000400030

Faria, D., B. Soares-Santos \& E. Sampaio. 2006. Bats from the Atlantic rainforest of southern Bahia, Brazil. Biota Neotropica 6(2): 1-13. https://doi.org/10.1590/S1676-06032006000200022

FeiJó, J.A. \& H.L. Nunes. 2010. Primeiro registro de Myotis nigricans (Schinz, 1821) para o estado do Rio Grande do Norte, nordeste do Brasil. Chiroptera Neotropical 16(1): 531-534. https:// chiroptera.unb.br/index.php/cn/article/view/40

Ferrarezzi, H. \& E.A. Gimenez. 1996. Systematic patterns and the evolution of feeding habits in Chiroptera (Archonta: Mammalia). JournalofComparativeBiology 1(3):75-94.

Ferreira, R.L., X. Prous, B.L. F. De Oliveira \& M. Souza-Silva. 2010. Fauna subterrânea do Estado do Rio Grande do Norte: Caracterização e impactos. Revista Brasileira de Espeleologia 1(1): 25-51. http://www.icmbio.gov.br/revistaeletronica/index.php/ RBEsp/article/view/70

Graciolli, G. \& C.J.B.D. Carvalho. 2001. Moscas ectoparasitas (Diptera, Hippoboscoidea, Nycteribiidae) de morcegos (Mammalia, Chiroptera) do Estado do Paraná, Brasil. I. Basilia, taxonomia e chave pictórica para as espécies. Revista Brasileira de Zoologia 18(1): 33-49. http://www.scielo.br/pdf/rbzool/v18s1/v18suplla02.pdf

Gregorín, R. \& L.D.F. Mendes.1999. Sobre quirópteros (Emballonuridae, Phyllostomidae, Natalidae) de duas cavernas da Chapada Diamantina, Bahia, Brasil. Iheringia, Série Zoologia 86: 121-124.

Gonçalves, E. \& R. GRegorin. 2004. Quirópteros da Estação Ecológica da Serra das Araras, Mato Grosso, Brasil, com o primeiro registro de Artibeus gnomus e A. anderseni para o cerrado. Lundiana 5(2): 143-149.

GorRenSEn P.A. \& M.R. WiLLIG. 2004. Landscape responses of bats to habitat fragmentation in Atlantic forest of Paraguay. Journal of Mammalogy 85(4): 688-697. https://doi.org/1.1644/BWG-125

Kasper, C.B., M.J.Feldens, F.D. Mazim, A. Schneider, C.V.CAdemARTORi \& H.C.Z. Grillo. 2007. Mamíferos do Vale do Taquari, região central do Rio Grande do Sul. Biociências 15(1): 53-62. http://revistaseletronicas.pucrs.br/ojs/index.php/fabio/article/ view/257/1742

LEAL, I.R. \& J.M.C. DA Silva. 2003. Ecologia e conservação da Caatinga. Editora Universitária da Universidade Federal de Pernambuco, 3rd edition. Recife, Pernambuco, Brazil. 797 pp.

Luz, J.L., L.D.M. Costa, E.C. Lourenço \& C.E.L. EsbÉRARD. 2011. Bats (Mammalia, Chiroptera) from Reserva Rio das Pedras, Rio de Janeiro, Southeastern Brazil. Biota Neotropica 11(1): 95-101. https://doi.org/10.1590/S1676-06032011000100009

Marques-Aguiar, S.A., M.V. Del Aguila, G.F. Aguiar, N. SaldaNHA, J.D.S. Silva-Junior \& M.M. Rocha. 2003. Caracterização e perspectivas de estudo dos quirópteros da Estação Científica Ferreira Penna-município de Melgaço-PA. Idéias e Debates, Belém 6: 1-3. \& \& \& Marques, R.V., V.C. Cristina \& M.P. Susi. 2011. Mastofauna no Planalto das Araucárias, Rio Grande do Sul, Brasil. Revista Brasileira de Biociências 9(3): 278-288. http:// www.ufrgs.br/seerbio/ojs/index.php/rbb/article/view/1414

Martins, A.C., E. Bernard \& R. Gregorin. 2006. Inventários biológicos rápidos de morcegos (Mammalia, Chiroptera) em três unidades de conservação do Amapá, Brasil. Revista Brasileira de Zoologia 23(4): 1175-1184. https://doi.org/10.1590/S010181752006000400026

Medellín, R.A. 1988. Prey of Chrotopterus auritus, with notes on feeding behavior. Journal of Mammalogy 69(4): 841-844. http:// www.jstor.org/stable/1381644

Medellín, R.A. 1989. Chrotopterus auritus. Mammalian Species 343: 1-5. https://doi.org/10.2307/3504232

MMA. 2017. Ministério do Meio Ambiente. Caatinga. Accessed at http://www.mma.gov.br/biomas/caatinga, 24 February 2017.

Modesto, T.C., F.S. PessôA, M.C. Enrici, N. Attias, T. JordãoNogueira, L.D.M. Costa, H.G. Albuquerque \& H.D.G. BerGALlo. 2008. Mamíferos do Parque Estadual do Desengano, Rio de Janeiro, Brasil. Biota Neotropica 8(4): 153-159. http://www. biotaneotropica.org.br/v8n4/pt/abstract?article+bn01408042008

Moratelli, R., D. Dias \& C.R. Bonvicino. 2010. Estrutura e análise zoogeográfica de uma taxocenose de morcegos no norte do Estado do Amazonas, Brasil. Chiroptera Neotropical 16(1): 661-671. http:// www.chiropteraneotropical.net/index.php/cn/article/view/254

Nobre, P.H., A.D.S. Rodrigues, I.D.A. Costa, A.E.D.S. Moreira 
\& H.H. Moreira. 2009. Similaridade da fauna de Chiroptera (Mammalia), da Serra Negra, municípios de Rio Preto e Santa Bárbara do Monte Verde, Minas Gerais, com outras localidades da Mata Atlântica. Biota Neotropica 9(3): 151-156. http://www. biotaneotropica.org.br/v9n3/pt/abstract?article+bn03309032009

Nogueira, M.N., A. Pol \& A.L. Peracchi. 1999. New records of bats from Brazil with a list of additional species for the chiropteran fauna of the state of Acre, western Amazon basin. Mammalia 63(3): 363-367. https://doi.org/10.1515/mamm.1999.63.3.363

Nogueira, M.R., Pol, A., Pessôa, L.M., J.A.D., Oliveira \& A.L. PERACCHI.2015. Small mammals (Chiroptera, Didelphimorphia, and Rodentia) from Jaíba, middle Rio São Francisco, northern Minas Gerais State, Brazil. Biota Neotropica 15(2): 1-18. https:/ doi.org/10.1590/1676-06032015012614

Oliveira, J.A., P.R. GonçAlves \& C.R. Bonvicino. 2003. Mamíferos da Caatinga; pp. 275-303, in: I.R. Leal, M. TABARELli \& J.M.C. Silva (eds.). Ecologia e Conservação da Caatinga. Recife: Universidade Federal de Pernambuco.

Oliveira, A.K.M., M.D. Oliveira, S. Favero, L.F. de Oliveira. 2011. Diversity, similarity and trophic guild of chiropterofauna in 3 southern Pantanal sub-regions, state of Mato Grosso do Sul, Brazil. BiologicalSciences 34(1): 33-39. https://doi.org/10.4025/ actascibiolsci.v34i1.7596

Oliveira, S.L, L.A.S. Souza, H.K. Silva \& K.C. Faria. 2015. Spatial configuration of the occurrence of bat species (Mammalia: Chiroptera) in eastern Mato Grosso, Brazil. Biota Neotropica 15(1): e20140122. https://doi.org/10.1590/1676-06032014012214

Ortêncio-Filho, H. \& N.R. Reis. 2009. Species richness and abundance of bats in fragments of the stationalsemidecidual forest, Upper Paraná River, southern Brazil. Brazilian Journal of Biology 69(2): 727-734. https://doi.org/10.1590/S1519-69842009 000300026

Paglia, A.P., G.A. da Fonseca, A.B. Rylands, G. Herrmann, L.M. Aguiar, A.G. Chiarello, Y.R.L. Leite, L.P. Costa, S. Siciliano, M.C.M. KierulfF, S.L. Mendes, V.C. Tavares, R.A. MitterMEIER \& J.M. PATTON. 2012. Lista anotada dos mamíferos do Brasil, $2^{\mathrm{a}}$ Edição. Annotated checklist of Brazilian mammals. Occasional papers in Conservation Biology 6: 76 pp.

Passos, F.C., W.R. Silva, W.A. Pedro \& M.R. Bonin. 2003. Frugivoría em morcegos (Mammalia, Chiroptera) no Parque Estadual Intervales, sudeste do Brasil. Revista Brasileira de Zoologia 20(3): 511-517. https://doi.org/10.1590/S0101-81752003000300024

Pedro, W.A., F.C. Passos \& B.K. Lim. 2014. Morcegos (Chiroptera: Mammalia) da Estação Ecológica dos Caetetus, estado de São Paulo. Chiroptera Neotropical 7(1-2): 136-140. http://www. chiropteraneotropical.net/index.php/cn/article/view/124

Pedro, W.A. \& V.A. TAdDeI. 2014. Bats from southwestern Minas Gerais, Brazil (Mammalia: Chiroptera). Chiroptera Neotropical 4(1): 85-88. http://www.chiropteraneotropical.net/index.php/ $\mathrm{cn} /$ article/view/179

Peracchi, A.L. \& S.T. Albuquerque. 1993. Quirópteros do município de Linhares, Estado do Espírito Santo, Brasil (Mammalia, Chiroptera). Revista Brasileira de Biologia 53(4): 575-581. http:// rl.ufrrj.br/labmasto/publicacoes/32.pdf

Peters, F.B., R.P.R. de O. Roth, L.F. Machado, E. De L. Coelho, D.M.H. Jung \& A.U. Christoff. 2010. Assembléia de mamíferos dos agroecossistemas constituintes da bacia hidrográfica do rio da Várzea, Rio Grande do Sul. Biotemas 23(4): 91-107. https://doi. org/10.5007/2175-7925.2010v23n4p91

Quintela, F.M., C. Ibarra, S.V.D. Oliveira, I.G. Medvedovisky, F. Correa, D. Gianuca, A. Gava \& S.M. Pacheco. 2011. Mammalia, Chiroptera, Rio Grande, state of Rio Grande do Sul, Brazil. Check List 7(4): 443-447. https://doi.org/10.15560/7.4.443

ReID, F. 2009. A field guide to the mammals of Central America and southeast Mexico. 2nd Edition. New York: Oxford University Press. 346 pp.
Reis, N.R. Dos., A.L. Peracchi \& M.L.Sekiama.1999. Morcegos da Fazenda Monte Alegre, Telêmaco Borba, Paraná (Mammalia, Chiroptera). Revista Brasileira de Zoologia 16 (2): 501-505. http:// www.scielo.br/pdf/rbzool/v16n2/v16n2a15

Reis, N.R, M.L.S. Barbieri, I.P. Lima \& A.L. Peracchi. 2003. O que é melhor para manter a riqueza de espécies de morcegos (Mammalia, Chiroptera): um fragmento florestal grande ou vários fragmentos de pequeno tamanho. Revista Brasileira de Zoologia 20(2): 225-230. https://doi.org/10.1590/S0101-81752003000200009

Rodrigues, F.H., L. Silveira, A.P. Carmignotto, A.M. Bezerra, D.C. Coelho, H. Garbogini, J. Pagnozzi, \& A. Hass. 2002. Composição e caracterização da fauna de mamíferos do Parques Nacional das Emas, Goiás, Brasil. Revista Brasileira de Zoologia 19(2): 589-600. https://doi.org/10.1590/S0101-81752002000200015

Sato, T.M., M.C. Carvalho-Ricardo, W. Uieda \& F.C. Passos. 2015. Estrutura da comunidade de morcegos (Mammalia, Chiroptera) da Estação Experimental de Itirapina, estado de São Paulo, Brasil. Papeis Avulsos Zoologia. 55(1): 1-11. https://doi. org/10.1590/0031-1049.2015.55.01

SÁ-Neto, R.J. \& J. Marinho-Filho. 2013. Bats in fragments of xeric woodland caatinga in Brazilian semiarid. Journal of Arid Environments 90: 88-94. https://doi.org/10.1016/j.jaridenv.2012.10.007

Sbragia, I.A. \& A. CARDoso. 2008. Quiropterofauna (Mammalia: Chiroptera) cavernícola da Chapada Diamantina, Bahia, Brasil. Chiroptera Neotropical 14(1): 360-365. https://chiroptera.unb. br/index.php/cn/article/view/107

Sikes, R.S. \& W.L. Gannon. 2011. Animal Care and Use Committee of the American Society of Mammalogists. Guidelines of the American Society of Mammalogists for the use of wild mammals in research. Journal of Mammalogy 92(1): 235-253. https://doi. org/10.1644/10-mamm-f-355.1

Silva, J.P.A., A.R. Carvalho \& J.A. De O. Motta. 2009. Fauna de morcegos (Mammalia, Chiroptera) em cavernas do bioma Cerrado na região de Indiara (Goiás). Revista Brasileira de Zoociências 11(3): 209-217. https://zoociencias.ufjf.emnuvens.com.br/ zoociencias/article/view/1259/995

Simmons, N.B. 2005. Order Chiroptera; pp. 312-529, in: D.E. Wilson \& D.M. REEDER (eds.). Mammal species of the world: a taxonomic and geographic reference. Baltimore: Johns Hopkins University Press.

Sousa, M.A.N., A. Langguth \& G.E. do Amaral. 2004. Mamíferos dos brejos de altitude Paraíba e Pernambuco; pp. 229-254, in: K.C. PôRto, J.J.P. CABral \& M. TABarelli (eds.). Brejos de altitude em Pernambuco e Paraíba: história natural, ecologia e conservação. Brasília: Ministério do Meio Ambiente. http://www. mma.gov.br/estruturas/chm/_arquivos/parte7_brejos.pdf

Stallings, J.R., G.A. DA FonseCA, L.P.D.S. Pinto, L.M.D.S., Aguiar, E.L. SÁbato. 1990. Mamíferos do Parque Florestal Estadual do Rio Doce, Minas Gerais, Brasil. Revista Brasileira de Zoologia 7(4): 663-677. https://doi.org/10.1590/S0101-81751990000400022

Tavares, V.C., L.M. Aguiar, F.A. Perini, F.C. Falcão \& R. GreGorín. 2010. Bats of the state of Minas Gerais, southeastern Brasil. Chiroptera Neotropical 16(1): 675-705. http://chiroptera neotropical.net/index.php/cn/article/view/257

Trajano, E. 1985. Ecologia de populações de morcegos cavernícolas em uma região cárstica do sudeste do Brasil. Revista Brasileira de Zoologia 2(5): 255-320. https://doi.org/10.1590/S0101-817519 84000100001

Uieda, W., T.M. Sato, M.C. de Carvalho \& V. Bonato. 2007. Fruits as unusual food items of the carnivorous bat Chrotopterus auritus (Mammalia, Phyllostomidae) from southeastern Brazil. Revista Brasileira Zoologia 24(3): 844-847. https://doi.org/10.1590/S010181752007000300035

Uieda, W., I. Sazima \& A. Storti Filho. 1980. Aspectos da biologia do morcego Furipterus horrens (Mammalia, Chiroptera, Furipteridae). Revista Brasileira de Biologia 40(1): 59-66. http:// 
repositorio.inpa.gov.br/handle/123/5284

Vizotto, L.D. \& V.A. TADDEI. 1973. Chave para determinação de quirópteros brasileiros. São José do Rio Preto: Editora da UNESP. $72 \mathrm{pp}$.

Williams, S.L. \& H.H. Genoways. 2008. Subfamily Phyllostominae Gray, 1825; pp. 255-300, in: A.L. GARDNER (ed.). Mammals of South America. Volume 1: marsupials, xenarthrans, shrews, and bats. Chicago and London: The University of Chicago Press, Chicago, IL.

Witt, A.A. \& M.E. Fabian. 2010. Hábitosalimentares e uso de abrigosporChrotopterus auritus (Chiroptera, Phyllostomidae). Mastozoología Neotropical 17(2): 353-360. http://ref.scielo.org/nsfdyv
Authors' contributions. GHNB contributed in collection of data and wrote manuscript; JPMA and JCVM contributed in collecting data in the field, revised and wrote part of the manuscript; PAR did cranial analysis, map and manuscript revision; MAFK revised the manuscript.

Received: 30 October 2016

Accepted: 31 March 2017

Academic editor: Annia Rodriguez-San Pedro 